Original Article $\quad$ www.pjkd.com.pk

\title{
Frequency and Microbiological Profile of Catheter-Related Infections in Hemodialysis Patients Receiving Gentamicin as Antimicrobial Lock Therapy for Prophylaxis
}

\author{
Sumbal Nasir Mahmood 1, Sabaa Asif', Muhammad Amim Anwar², Osama \\ Kunwer Naveed ${ }^{3}$ \\ ${ }^{1}$ Department of Nephrology, Ziauddin Medical University, Karachi, Pakistan. \\ 2Department of Anesthesiology, Liaquat National Hospital, Karachi, Pakistan \\ ${ }^{3}$ Dow Medical College, University of Health Sciences, Karachi, Pakistan
}

\begin{abstract}
Background and Objective: Use of Tunneled cuffed catheters (TCC) and non tunneled catheters are commonly used as an access for hemodialysis (HD). They remain a source of infection in this patient population and results in significant morbidity and mortality, and poses a great financial burden on health care system.

Antibiotic lock solution (ALS) in the lumen of catheter is a mean to decrease the possibility of catheter related blood stream infection (CRBSI). We conducted this study using Gentamicin as ALS to observe the frequency of catheter related infections (CRI) including CRBSI and exit site infection in this hemodialysis patients being dialyzed through tunneled and temporary hemodialysis catheters.
\end{abstract}

Methods This descriptive, cross sectional study was conducted at Dr. Ziauddin Hospital, North Campus between January 2017December 2017.

Symptomatic patients for CRBSI were included in this study. Blood culture from the catheter ports and periphery and exit site swab if pus visible were sent for culture sensitivity. Actual frequencies and comorbid conditions effect was analyzed.

Results Total 138 patients of either gender with age more than 18 years to less than 80 years were evaluated. $44.9 \%$ were male and mean age of patients was $58.79 \pm 14.05$ years. $70.3 \%$ patients were diabetic for $15.61 \pm 4.61$ years and $11.6 \%$ were using immunosuppressive drugs.

\begin{abstract}
The overall mean catheter duration was $22.27 \pm 14.39$ days and mean hemodialysis duration was $25.32 \pm 44.31$ weeks. Tunneled catheter was placed in $40.6 \%$ and $46.4 \%$ had femoral temporary catheters. Blood culture was done for 51 cases and $21.5 \%$ were positive. Tip culture was positive in 33.3\% patients. No significant association of catheter related infections with gender, age, catheter duration, catheter type, catheter site and diabetes mellitus was found, $p>0.05$. Microbiological profile data showed a predominance of Grampositive organisms. In our study, $23.18 \%$ patients were found to have catheter related infections, out of which $61.5 \%$ were found with gentamicin resistance including colonization.
\end{abstract}

Conclusion We report a high frequency of catheter related infections despite using ALS as prophylaxis, with predominant organisms being gram positives and a high incidence of gentamycin resistance.

Keywords: Catheter related infections, antibiotic lock solution, Gentamycin, Hemodialysis, frequency

Corresponding Author:

Dr. Sumbal Nasir Mahmood

Professor \& HOD

Department of Nephrology

Ziauddin Medical University, Karachi, Pakistan

Email: sumbaloo@yahoo.com

Received: April 26, 2020. Accepted June 24, 2020.

PJKD 2020;4(3):284-289

\section{Introduction:}

Use of double lumen catheters (both tunneled and non-tunneled) as an access for hemodialysis (HD) remains common worldwide. Despite multiple guidelines to avoid its use in patients anticipated to start HD non cuffed catheters remain the main source of vascular access in patients requiring acute HD, and hence are indispensable. Latest data in US reported about $80 \%$ of patients initiate chronic HD via a tunneled cuff dual lumen catheter. ${ }^{1,2}$ 
These catheters can be used for short term basis as a bridge for maturity of arteriovenous fistula (AVF) or may be used as permanent access in those who do not have alternative options, like those with low ejection fraction or poor vessels in which AVF or AV grafts are not possible.

Both tunneled cuffed catheters (TCC) and non-cuffed catheters have complications, with infections being the most prevalent that include exit site infection, tunnel infections and catheter related blood stream infection (CRBSI). ${ }^{4.7}$ Other infectious complications that are life threatening include endocarditis, lung abscess, osteomyelitis and endophthalmitis. ${ }^{8}$ Non infectious complications include central venous stenosis and catheter thrombosis.

Since catheter related infections (CRI) poses a great risk both in terms of morbidity and mortality it also has a substantial burden on cost to the health care system. According to US renal data system, infection is the most leading cause of death on patients with end stage renal disease (ESRD) and leading cause of catheter removal and morbidity in dialysis patients. ${ }^{9-12}$

Several modalities including intraluminal and extraluminal approaches have been assessed as a means to prevent CRI, one of which is to instill an antimicrobial solution (ALS) into the lumen of catheter to avoid intra luminal source of infection, as these solutions can prevent biofilm formation which constitutes a permanent source of bacteremia. ${ }^{13}$

Jaffer et al. meta analyzed several RCT in patients with HD with catheters and showed that ALS had a positive trend in reducing frequency of CRI without producing significant side effects. ${ }^{14}$ The available ALS include Gentamycin, Vancomycin, Cefotaxime and Cefazolin beside others, in combination with citrate or heparin. Clinical practice guidelines recommend ALS therapy for better prevention and treatment of CRI. ${ }^{15,16}$

\section{Objective:}

We conducted this descriptive, cross sectional study to a) see the frequency of CRI including CRBSI, exit site infection and catheter tip infections in patients undergoing HD via tunneled or non-tunneled catheters receiving Gentamycin as ALS as prophylaxis and b). the microbiological profiles of patients developing these infections. In addition, incidence of gentamycin resistance was also observed.

\section{Methods}

This descriptive, cross sectional study was conducted at Dr. Ziauddin Hospital, North Campus between January 2017- December 2017.

Inclusion Criteria. All adult patients more than 18 years and less than 80 years of age, both males and females undergoing HD via tunneled catheter inserted as a bridge for maturity of AVF or via non tunneled temporary HD catheter inserted for acute HD after informed consent were enrolled in the study.

Exclusion Criteria: Refusal to participate in the study, those with bacteremia from known alternate sites and patients with previous history of intravenous drug abuse, chronic skin wounds, endocarditis and HIV infection were excluded. Patients who had tunneled catheters inserted as permanent access were also excluded.

All patients received gentamycin as ALS on Day 1 of insertion of catheter and after every HD session in the following manner in tunneled-catheters: 5,000 international units of heparin with $10 \mathrm{mg}$ gentamicin in $1 \mathrm{ml} 0.9 \%$ saline.

In non-tunneled catheters: 10,000 international units of heparin with $20 \mathrm{mg}$ gentamicin in $2 \mathrm{ml} 0.9 \%$ saline.

Data was collected for signs of infection if patients developed fever (temperature more than 100

${ }^{0} \mathrm{~F}$ ), shivering, unexplained tachycardia or hypotension (mean arterial blood pressure $<65 \mathrm{mmHg}$ ), and blood culture both from periphery and catheter port was taken. In addition, culture of the catheter tip was sent if catheter was removed either because of persistent infective signs or routine removal of catheter once indication of HD via catheter was terminated. CRBSI was defined as any positive blood or catheter tip growing an organism, with colony counts of $>10^{5}$ colony forming units/ml along with symptoms of infection.

Colonization was defined as any catheter tip growing significant organisms without clinical signs of infection.

Exit site infection was defined as positive pus culture taken from insertion site of catheter.

Data was recorded for age, gender, type of catheter (tunnelled or non-tunnelled), duration of the use of catheter, site of catheter placement (femoral, internal jugular or subclavian), duration on hemodialysis, diabetes mellitus, immunosuppressive drugs, duration of use of immunosuppressive drugs, and any other source of infection.

Culture was performed using MacConkey agar and Antibiotic Disc Diffusion method was used for testing for antibiotic sensitivity. Catheter tips, in cases where they were removed, were cultured using Mannitol Salt Agar (MSA) or Sheep Blood Agar (SBA). In patients with exit-site purulent discharge, pus culture was taken using a sterile swab and culture was performed using MacConkey agar and Antibiotic Disc Diffusion method was used for testing for antibiotic sensitivity.

\section{Statistical Analysis:}

Data was analyzed using SPSS version 23.0 (Armonk, New York, USA).

Mean and standard deviation was calculated for quantitative variables like age, duration of use of catheter. Frequency and percentage were calculated for qualitative variables like gender, catheter-related infection, and diabetes mellitus.

Effect modifiers like age, gender, duration of use of catheter, duration of diabetes, duration of immunosuppressive drugs, site of catheter and type of catheter was controlled through stratification. Post-stratification Chi-square test was applied by taking p-value less than 0.05 as significant 


\section{Results}

Total 138 patients of either gender with age more than 18 years to less than 80 years meeting inclusion criteria of study were evaluated. Demographic data is presented in Table 1. Mean age of patients was $58.79 \pm 14.05$ years .

Table - 1: Baseline characteristics of 138 patients undergoing hemodialysis using temporary and tunneled hemodialysis catheter.

\begin{tabular}{|l|c|}
\hline Characteristics & Frequency (\%) \\
\hline Age & $58.79+/-14.05$ \\
\hline Age >60 years & $67(49)$ \\
\hline Age <60 years & $71(51)$ \\
\hline Male / Female & $62(44.9) / 76(55.1)$ \\
\hline Diabetics Mellitus \& Duration (Years) & $97(70.3) / 15.61+/-4.61$ \\
\hline Immunosuppression & $16(11.6)$ \\
\hline Catheter Duration (Days) & $22.27+/-14.39$ \\
\hline Hemodialysis Duration (Days) & $25.32+/-44.31$ \\
\hline
\end{tabular}

Majority of the patients $70.3 \%$ patients were diabetic with mean duration $15.61 \pm 4.61$ years Among diabetic patients, $36.1 \%$ had good control of DM with $\mathrm{HbAlc}<7$. Immunosuppressive drugs were found positive for $11.6 \%$ patients with mean usage duration $26.25 \pm 21.56$ days.

The overall mean catheter duration was $22.27 \pm 14.39$ days and mean hemodialysis duration was $25.32 \pm 44.31$ weeks as shown in Table 1 and Table 2 describes details of catheter characteristics.

Table 2: Type of hemodialysis catheters and its duration among 138 patients undergoing hemodialysis.

\begin{tabular}{|c|c|}
\hline Catheter Characteristics & Frequency (\%) \\
\hline $\begin{array}{c}\text { Catheter type } \\
\text { Tunneled / Non-Tunneled }\end{array}$ & $56(40.6) / 82(59.4)$ \\
\hline $\begin{array}{c}\text { Duration of Catheter (Days) } \\
<14 />14\end{array}$ & $62\left(45^{*}\right) / 76\left(55^{*}\right)$ \\
\hline $\begin{array}{c}\text { Site } \\
\text { Femoral / Internal Jugular }\end{array}$ & $64(46.4) / 74(53.6)$ \\
\hline
\end{tabular}

*Days

Blood culture was done for 51 cases, out of which $21.5 \%$ were found positive. Tip culture was done for 90 cases, out of which $33.3 \%$ were found positive. Further evaluation of data found that out of 30 cases positive for tip culture only 10 (11.1\%) were positive in symptomatic patients and the rest $20(22.2 \%)$ were positive in patients in which tip was sent as routine once the catheter was removed and hence was considered colonization.

Microbiological profile data showed a predominance of Gram-positive organisms with $36 / 52$ (69.23\%) being gram positive and 16/52 $(30.76 \%)$ gram negative organisms. Details of the organisms are reported in Table 3. In our study, 23.18\% patients were found to have catheter related infections, out of which $61.5 \%$ were found with gentamicin resistance including colonization. (Table 3). Pus culture was done for 11 cases and all were found positive as described in Table 4.

Stratification with respect to gender, age, catheter duration, catheter type, catheter site and diabetes mellitus was done to observe effect of these modifiers on catheter related infections. P-value $\leq 0.05$ was considered as significant.

Table 3: Microbiological profile of organisms isolated in 52 of 138 patients undergoing hemodialysis via temporary or permanent catheter.

\begin{tabular}{|c|c|c|c|c|c|}
\hline Microorganism & Blood Culture & Exit site & $\begin{array}{c}\text { Catheter } \\
\text { tip(symptomatic) }\end{array}$ & $\begin{array}{c}\text { Catheter tip } \\
\text { (asymptomatic) }\end{array}$ & $\begin{array}{c}\text { Resistance to } \\
\text { Gentamycin }\end{array}$ \\
\hline Coagulase negative Staph & 5 & 3 & 4 & 8 & 14 \\
\hline MRSA & 2 & 3 & 3 & 6 & 8 \\
\hline Klebsiella & 1 & 2 & 2 & 3 & 5 \\
\hline VRE & 2 & 0 & 0 & 0 & 1 \\
\hline Pseudomonas & 0 & 3 & 0 & 2 & 3 \\
\hline Acinetobacter & 1 & 0 & 0 & 1 & 0 \\
\hline E. coli & 0 & 0 & 1 & 20 & 32 \\
\hline TOTAL & 11 & 11 & 10 & & 1 \\
\hline
\end{tabular}


The results showed insignificant association of catheter related infections with gender $(\mathrm{p}=0.404)$, age $(\mathrm{p}=0.187)$, catheter duration $(\mathrm{p}=0.630)$, catheter type $(\mathrm{p}=0.748)$, catheter site $(\mathrm{p}=0.147)$ and diabetes mellitus $(\mathrm{p}=0.551)$ as shown in Table 5

Table 4: Frequency distribution of cultures obtained for suspected infections in 138 patients undergoing maintenance hemodialysis via tunneled and temporary venous catheters.

\begin{tabular}{|c|c|c|c|l|}
\hline \multicolumn{2}{|c|}{ Source } & Positive & Negative & Total \\
\hline \multicolumn{2}{|c|}{ Blood } & 11 & 40 & $11 / 51(21.5)$ \\
\hline \multicolumn{2}{|c|}{ Pus } & 11 & 0 & $11 / 11(100)$ \\
\hline \multirow{2}{*}{ Catheter Tip } & Symptomatic & 10 & & $10 / 90(11.1)$ \\
\cline { 2 - 3 } & Asymptomatic & 20 & & 20 \\
\cline { 5 - 6 } & & & & $20 / 90(22.2)$ \\
\hline
\end{tabular}

\section{Discussion}

To our knowledge this is the third study from Pakistan to obtain frequency of catheter related infections and only study to detail their microbiological profiles in patients receiving ALS. We did not find any significant association of catheter related infections with gender, age, catheter duration, catheter type, catheter site, and diabetes mellitus.

The earlier two studies using Gentamicin similar to our study however found a significant prevention of CRI with ALS. ${ }^{17,18}$ The earlier studies were comparative studies whereas we here report the frequency of CRI as a cross sectional study. We have reported the microbiological profile whereas the other studies by Kashif et.al. and Shabaz et.al., only reported being positive or negative. ${ }^{17,18}$ We have also shown a higher rate of Gentamicin resistance in 32/52 (61\%) of CRI. Furthermore, 18/52 (35\%) had culture positive for gram negative organisms and VRE.

Table 5: Chi Square test applied on Patient and catheter characteristics influence on CRI in 138 hemodialysis patients,

\begin{tabular}{|c|c|c|c|c|c|}
\hline \multirow{2}{*}{ Patient and catheter characteristics } & \multicolumn{2}{|c|}{ Catheter related infection } & \multirow{2}{*}{ Total } & \multirow{2}{*}{ P-Value } \\
\cline { 3 - 5 } & Male & $21(33.9)$ & $41(66.1)$ & 62 & \\
\hline \multirow{2}{*}{ Gender } & Female & $31(40.8)$ & $45(59.2)$ & 76 & \multirow{2}{*}{$0.404^{* *}$} \\
\hline & Total & 52 & 86 & 138 & \\
\hline Age & $<60$ & $23(32.4)$ & $48(67.6)$ & 71 & \\
\hline & $>60$ & $29(43.3)$ & $38(56.7)$ & 67 & \multirow{2}{*}{$0.187^{* *}$} \\
\hline & Total & 52 & 86 & 138 & \\
\hline Catheter duration & $<14$ days & $22(35.5)$ & $40(64.5)$ & 62 & \\
\hline & $>14$ Days & $30(39.5)$ & $46(60.5)$ & 76 & \multirow{2}{*}{$0.630^{* *}$} \\
\hline & Total & 52 & 86 & 138 & \\
\hline Catheter type & Tunneled & $22(39.3)$ & $34(60.7)$ & 56 & \\
\hline & Non-Tunneled & $30(36.6)$ & $52(63.4)$ & 82 & \multirow{2}{*}{$0.748^{* *}$} \\
\hline & Total & 52 & 86 & 138 & \\
\hline Catheter site & Femoral & $20(31.3)$ & $44(68.8)$ & 64 & \\
\hline & Internal jugular & $32(43.2)$ & $42(56.8)$ & 74 & \multirow{2}{*}{$0.147^{* *}$} \\
\hline & Total & 52 & 86 & 138 & \\
\hline
\end{tabular}

**Not Significant at $\mathrm{p}$ value of $<0.05$ levels

Our study did not find any correlation to catheter duration and diabetes which is contradictory to published data in which these two variables have been associated with risks of CRBSI as reported by F. Sahli et al and Lemaire et al., however their study was in patients who did not receive any ALS, whether ALS is helpful in these groups cannot be commented as we do not have controls and our study population is limited study..$^{19,20}$ 
Our study is in accordance with data provided by Nabi et al , and others where catheter site whether femoral or internal jugular had no correlation to catheter related infections. ${ }^{2122}$

We report a high frequency of gram-positive organisms in our study with Coagulase negative Staphylococcus and Methicillin Resistant Staph. Aureus dominating the profile as well as a high incidence of gentamycin resistance with nearly $70 \%$ of organisms were resistant to it.

A similar study by Landry et al. was conducted which aimed at assessment of the long-term consequences of a gentamicin and heparin lock (GHL) protocol in maintenance hemodialysis patients using a TCC and to document the associated emergence of gentamicinresistant bacteremia in these patients. ${ }^{23}$ For all patients receiving hemodialysis during the 4-year study period (TCC and AV access), there were 131 episodes of bacteremia in 113 patients. In their study, a total of 121 Gram-positive bacteria (including 48Staphylococcus aureus, 37 coagulase-negativeStaphylococcus, and 17Enterococcus faecalis) and 17 Gram-negative organisms (most common being E. coli) were identified by blood culture. In terms of Gentamicin resistance, the Gram-positive group had 24 instances of resistance organisms with the most common being coagulase-negativeStaphylococcus followed by Faecalis. ${ }^{7.13}$ In the gram-negative group, no incidence of gentamicin resistance was observed in this study.

A single-center observational study from New Zealand found a trend toward increasing gentamicin resistance among coagulasenegative Staphylococci isolated from hemodialysis patients with a TCC receiving a GHL. ${ }^{24}$

There are no RCTs comparing the frequency of catheter related bacteremia with tunneled or non-tunneled HD catheters; although some previous prospective nonrandomized studies of $\mathrm{HD}$ patients observed a catheter-related bacteremia rate that was 2- to 3-fold greater for non-tunneled compared with tunneled $\mathrm{CVC}{ }^{25} \mathrm{~A}$ latest multicenter study of incident $\mathrm{HD}$ patients found that, compared with patients with $\mathrm{AVFs}$, the age-adjusted relative risk for developing a bloodstream infection in patients with arteriovenous grafts was 1.69 (95\% CI, 0.42-6.79); with tunneled CVC, 9.78 (95\% CI, 3.53-27.11) and with no tunneled CVC, 10.54 (95\% CI, 3.6928.20). ${ }^{26}$ Therefore, using tunneled or no tunneled dialysis catheters, skin flora can be introduced into the lumen during manipulation of the catheter and form a biofilm, which serves as a nidus for catheter related bacteremia. These results are almost similar to our study where we are seeing an overall nearly $20 \%$ infection rate with these catheters. Having a tunneled catheter in $41 \%$ of our patients had an impact on microbiological profile is speculative. The handling of tunneled catheters in our local circumstances need further evaluation and lack of strict protocol implementation is speculative. A separate study in tunneled hemodialysis catheters for evaluation of these factors and frequency of CRI is needed since more of our patients with tunneled catheter had CRI 39\% vs 36.6\%, Table 5.

The use of an alternative, nonantibiotic, catheter lock is a possible solution to prevent CRI in chronic hemodialysis patients with a TCC. Unfortunately, currently available alternative locks all have ongoing questions and concerns beyond their rather limited study data. Taurolidine with $4 \%$ sodium citrate has been shown to be effective in several studies but may be associated with an increase in catheter thrombosis and is not currently available in the United States. ${ }^{2728}$ High-dose citrate (30 to 47\%) has been well studied but is complicated by the rare but fatal risk of hypocalcemia that led to the FDA's recall of $47 \%$ citrate in $2000 .^{29}$

In a recent Cochrane review by Arechabal et.al. noted a significant benefit of ALS compared to the non-antibiotic lock solution or standard catheter lock with Heparin. ${ }^{30}$ Our study in comparison to this and other studies have shown a high frequency of CRI even with ALS possibly due to large number of gentamicin resistance in our patients. A detailed analysis and further local studies are needed to document the actual prevalence of antibiotic resistance among our maintenance hemodialysis patients.

The major limitation of our study is the small sample size and lack of control group so whether ALS is helpful in reducing infection rates cannot be commented.

\section{Conclusion}

Our study showed high frequency of catheter related infection and colonization. A high rate of gentamicin resistance was also noted among our patients. We suggest a randomized controlled study comparing ALS and standard catheter lock solution in MHD patients. Furthermore, local data on antimicrobial resistance patterns are needed to plan such studies.

Conflict of Interest : The authors do not report any conflict of interest or source of funding.

\section{References:}

1. Hemodialysis Adequacy 2006 Work Group. Clinical practice guidelines for hemodialysis adequacy. Update 2006. Am J Kidney Dis 2006: 48 Suppl 1; S2

2. United States Renal Data System. 2018 USRDS annual data report: Epidemiology of kidney disease in the United States. National institutes of Health, National Institutes of Diabetes and Digestive and K idney Diseases, Bethesda, MD 2018

3. Ramon Roca-Tey . Permanent Arteriovenous Fistula or Catheter Dialysis for Heart Failure Patients J Vasc Access.2016 Mar, https//; doi.org/10.5301/jva.500 Kairaitis LK, Gottlieb T. Outcome and complications of temporary haemodialysis catheters. Nephrol Dial Transplant 1999;14:1710 - 14.

4. Oliver MJ, Callery SM, Thorpe KE. Risk of bacteremia from temporary hemodialysis catheters by site of insertion and duration of use: a prospective study. Kidney Int 2000;58:2543 - 5.

5. Develter W, De Cubber A, Van Biesen W. Survival and complications of indwelling venous catheters for permanent use in hemodialysis patients. Artif Organs 2005;29:399 - 405. 
6. Stevenson KB, Adcox MJ, Mallea MC. Standardized surveillance of hemodialysis vascular access infections: 18-month experience at an outpatient, multifacility hemodialysis center. Infect Control Hosp Epidemiol 2000;21:200 - 3.

7. Weijmere MC, Vervloet MG, ter Wee PM. Compared to tunnelled cuffed haemodialysis catheters, temporary untunnelled catheters are associated with more complications already within 2 weeks of use. Nephrol Dial Transplant 2004;19:670 - 7.

8. O’ Grady NP, Alexander M, Dellinger EP, Gerberding JL, Heard SO, Maki DG, Masur H, McCormick RD, Mermel LA, Parson ML, Raad II, Randolph A, Weinstein RA(2002) Guidelines for the prevention of intravascular catheter related infections. MMWR Recomm Rep 51: 1-29

9. Rabindranath KS, Bansal T, Adams J, Systematic review of antimicrobials for the prevention of haemodialysis catheter-related infections. Nephrol Dial Transplant 2009;24:3763 - 74

10. Marr KA, Sexton DJ, Conlon PJ, Catheter-related bacteremia and outcome of attempted catheter salvage in patients undergoing hemodialysis. Ann Intern Med 1997;127:275 - 80.

11. Butterly DW, Schwab SJ. Dialysis access infections. Curr Opin Nephrol Hypertens 2000;9:631 - 5.

12. Mermel LA. Prevention of intravascular catheter-related infections. Ann Intern Med 2000;132:391 - 402.

13. Donlan RM. Biofilm formation: a clinically relevant microbiologic process. Clin Infect Dis 2001;33:1387 - 92.

14. Jaffer Y, Selby NM, Taal MW, A meta-analysis of hemodialysis catheter locking solutions in the prevention of catheter-related infection. Am J Kidney Dis 2008;51:233 - 41 .

15. Centers for Disease Control and Prevention (CDC). Guidelines for the Prevention of Intravascular Catheter-Related Infections, 2011. Atlanta, GA: Centers for Disease Control and Prevention; 2011. Available from: http://www.cdc.gov/hicpac/pdf/guidelines/bsi-guidelines2011.pdf. Accessed May 10, 2014.

16. Mermel LA, Allon M, Bouza E, Clinical practice guidelines for the diagnosis and management of intravascular catheter-related infection: 2009 Update by the Infectious Diseases Society of America. Clin Infect Dis. 2009;49(1):1 - 45.

17. Rafique K, Sabir O, Riaz MM, Rizvi N, Tarif N. Comparing the efficacy of Gentamicin-heparin solution to Heparin alone as catheter related blood stream infection in patients undergoing hemodialysis. Pak J Kidney Dis 2019;(4):67-73

18. Shahbaz M, Muhammad T, Umar. Prevention of catheter related blood stream infection using Heparin plus Gentamicin catheter lock solution in comparison to Heparin alone. A randomized controlled study. Pak J Kidney Dis 2019;(4):59-66

19. Nabi Z, Anwar S, Barhamein M, Al Mukdad H, El Nassri A. Catheter related infections in hemodialysis patients. Saudi J Kidney Dis Transpl 2009;20

20. Sahli F. Feidjel R. Laalaoui R.,Hemodialysis catheter- related infection;rates, risk factors and pathogens.http://dx.doi.org/10.1016/j.jiph.2016.06.008

21. Lemaire X, Morena M. Leray Moragues H, Henriet Viprey D, Chenine L, Defez-Fougeron C, et al. Analysis of risk factors for catheter-r elated bacteremia in 2000 permanent dual catheters for hemodialysis. Blood Purif 2009;28; 21-8.

22. Parienti JJ, Thirion M. Megarbane B. Souweine B. Ouchikhe A, Potito A et al. Femoral vs Jugularvenous catheterization and risk $f$ nosocomial events in adults requiring acute renal replacement therapy; a randomized controlled trial. JAMA 2008;299(20); 2413-22

23. Landry DL, Braden GL, Gobeille SL. Emergence of gentamicin resistant bacteremia in hemodialysis patients receiving gentamicin lock catheter prophylaxis. Clin J Am Soc Nephrol 2010; 5: 1799-1804

24. Abbas SA, Haloob IA, Taylor SL. Curry EM, King BB, Van Der Merwe WM et al. Effect of antimicrobial locks for tunneled hemodialysis catheters on bloodstream infection and bacterial resistance: a quality improvement report. Am J Kidney Dis. 2009;53(3): 492-502

25. Stevenson KB. Hannah EL, Lowder CA. Epidemiology of hemodialysis vascular access infections from longitudinal infection surveillance data; predicting the impact of NKF-DOQI clinical practice guidelines for vascular access. Am J Kidney Dis. 2002;39(3);549-555

26. Taylor G, Gravel D. Johnston L. Canadian Hospital Epidemiology Committee. Incidence of bloodstream infection in multicenter inception cohorts of hemodialysis patients. Am J Infect Control.2004;32(3); 155-160

27. Taylor C, Cahill J, Gerrish M, Little J: A new hemodialysis catheter locking agents reduces infections in hemodialysis patients. J Renal Care 2010; 34: 116-120

28. Allon M: Dialysis catheter -related bacteremia: Treatment an prophylaxis. Am J Kidney Dis 2004;44;779-791

29. Vendito M, Tezenas du Montcel S, Robert J, Trystam D et al. Effect of catheter- lock solutions on catheter- related infections and inflammatory syndrome in hemodialysis patients ; Heparin versus citrate 46\% versus heparin/gentamycin. Blood Purif 2010;29; 268-273

30. Arechabala MC, Catoni MI, Claro JC, Rojas NP, Rubio ME,Calvo MA. Antimicrobial Lock Solutions for Preventing Catheter-Related Infections in Haemodialysis. Cochrane Database Syst Rev.2018;4(4):CD010597. 